\title{
Top Physics at ATLAS
}

\author{
Marino Romano*i \\ INFN Bologna \\ E-mail: romano@bo.infn.it
}

This paper is an overview of recent results on top-quark physics obtained by the ATLAS collaboration from the analysis of $p p$ collisions at $\sqrt{s}=7$ and $8 \mathrm{TeV}$ at the Large Hadron Collider. Total and differential top-quark pair cross-section, single top cross-section and mass measurements are presented.

The XXI International Workshop High Energy Physics and Quantum Field Theory,

June 23 - June 30, 2013

Saint Petersburg Area, Russia

\footnotetext{
${ }^{*}$ Speaker.

${ }^{\dagger}$ On behalf of the ATLAS collaboration
} 


\section{Introduction}

The top quark is the heaviest quark, significantly heavier than its partner, the bottom quark. Once the bottom quark was experimentally discovered in 1977, the existence of a charge-2/3 quark in the third quark generation was expected to preserve the Standard Model (SM) renormalizability. The top quark was eventually discovered by both the CDF [1] and D0 [2] collaborations in 1995 at the Tevatron collider.

The top quark is special not only due to its large mass, but also due to its short lifetime which prevents it from hadronizing before decaying, i.e. there are no bound state hadrons made of top quarks. This allows to experimentally test the properties of the bare top quark itself through its decay products without diluting information in the hadronization process.

As the properties of the top quark are precisely predicted by the SM, top quark physics provides a sensitive probe to test the validity of the SM and a tool to investigate the Higgs boson properites and to potentially discover physics beyond the SM.

This overview will describe some of the recent measurements of top-pair and single-top production cross section and of top-quark mass, covering only a selection of the top physics results achieved by the ATLAS Collaboration using data collected in 2011 (with an integrated luminosity $\mathscr{L}=4.7 \mathrm{fb}^{-1}$ at $\left.\sqrt{s}=7 \mathrm{TeV}\right)$ and in $2012\left(\mathscr{L}=21.7 \mathrm{fb}^{-1}\right.$ at $\left.\sqrt{s}=8 \mathrm{TeV}\right)$. An updated and more complete list is available at the ATLAS public top result website [3]. The ATLAS detector and its performance are described in [4].

\section{Combination of the top pair cross-section measurements at $\sqrt{\mathbf{s}}=7 \mathrm{TeV}$}

A statistical combination of the top quark pair production cross-section at $\sqrt{s}=7 \mathrm{TeV}$ measurements has been performed using dilepton and single-lepton final states with $\mathscr{L}=0.70 \mathrm{fb}^{-1}$ and all-hadronic final states with $\mathscr{L}=1.02 \mathrm{fb}^{-1}$ [5].

For each channel a likelihood is defined as a function of the signal cross-section $\sigma_{t \bar{t}}$, the integrated luminosity $\mathscr{L}$, and several nuisance parameters $\alpha_{j}$ that correspond to various sources of systematic uncertainty. The full combination is then implemented as a product of the individual likelihoods of the component analyses. When forming the six-measurement combined likelihood, constraint terms for systematic uncertainties that are common across analyses are included only once. For each channel a profile likelihood is defined as

$$
\lambda\left(\sigma_{t \bar{t}}\right)=\frac{L\left(\sigma_{t \bar{t}}, \hat{\hat{\mathscr{L}}}, \hat{\hat{\alpha}}_{j}\right)}{L\left(\hat{\sigma}_{t \bar{t}}, \hat{\mathscr{L}}, \hat{\alpha}_{j}\right)},
$$

where $\hat{\sigma}_{t \bar{t}}, \hat{\mathscr{L}}, \hat{\alpha}_{j}$ denote the maximum likelihood estimate of all the parameters and $\hat{\hat{\mathscr{L}}}, \hat{\hat{\alpha}}_{j}$ represent the conditional maximum likelihood estimates of $\mathscr{L}$ and $\alpha_{j}$ fixing $\sigma_{t \bar{t}}$. The best fit value of the cross-section is $\sigma_{t \bar{t}}$ and the $68 \%$ confidence interval is derived from the values of $\sigma_{t \bar{t}}$ which give $-2 \log \lambda\left(\sigma_{t \bar{t}}\right)=1$. The combined measurement is

$$
\left.\left.\sigma_{t \bar{t}}=177 \pm 3 \text { (stat. }\right)_{-7}^{+8} \text { (syst. }\right) \pm 7 \text { (lumi.) } \mathrm{pb}=177_{-10}^{+11} \mathrm{pb},
$$

in good agreement with the theoretical prediction $\sigma_{t \bar{t}}^{\text {theory }}=165_{-16}^{+11} \mathrm{pb}$ obtained with approximate NNLO QCD calculations with HATHOR [7], as shown in Fig. 1. 


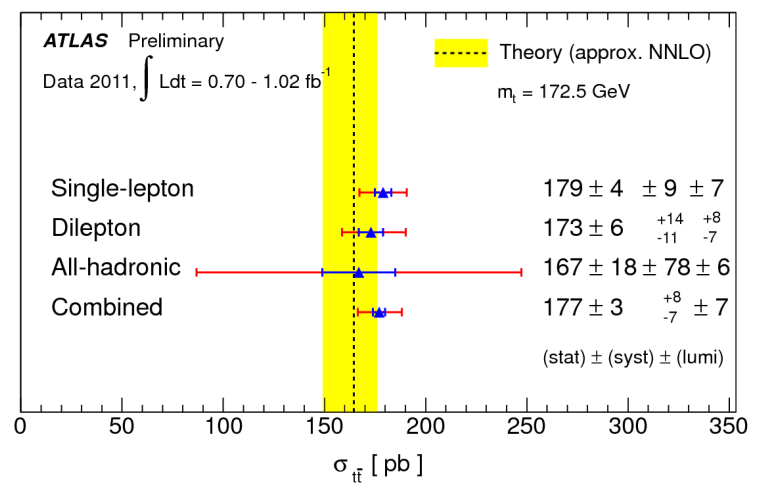

Figure 1: The measured value of $\sigma_{t \bar{t}}$ in the single-lepton channel, the dilepton channel, the allhadronic channel, and the combination of these three measurements.

\section{Top pair cross-section measurement at $\sqrt{\mathbf{s}}=8 \mathrm{TeV}$ in the lepton+jets channel}

At $\sqrt{s}=8 \mathrm{TeV}$, the top-antitop production cross-section has been measured in the lepton+jets channel in a dataset corresponding to an integrated luminosity of $5.8 \mathrm{fb}^{-1}$ [6]. In this measurement, the number of $t \bar{t}$ candidates has been extracted from the data using a likelihood discriminant template fit. The discriminant for the $i$-th event is defined as the ratio of the signal likelihood $\mathscr{L}_{i}^{s}$ to the sum of the signal and background likelihood $\mathscr{L}_{i}^{s}+\mathscr{L}_{i}^{b}$. Simulated $t \bar{t}$ and $W+$ jets events are used for the signal and background likelihood functions, respectively. The variables used in the likelihood are the lepton pseudorapidity $\eta_{l}$ and the transformed event aplanarity $A^{\prime}=\exp (-8 A){ }^{1}$ Likelihood discriminant functions are then used to produce binned distributions (templates) for the $t \bar{t}$ signal and each of the backgrounds.

The number of observed events $N_{t \bar{t}}$ is obtained by means of a likelihood fit of the observed likelihood discriminant to the the sum of the signal and background templates. The $t \bar{t}$ production cross-section is calculated as

$$
\sigma_{t \bar{t}}=\frac{N_{t \bar{t}}}{\mathscr{L} \cdot B R \cdot \varepsilon_{s i g}}
$$

where $\mathscr{L}$ is the integrated luminosity, $B R$ the combined branching ratio for dileptonic and semileptonic $t \bar{t}$ decays, and $\varepsilon_{\text {sig }}$ the product of the signal acceptance and efficiency calculated on a $t \bar{t}$ sample without fully-hadronic decays. The cross-section is found to be

$$
\sigma_{t \bar{t}}=241 \pm 2 \text { (stat.) } \pm 31 \text { (syst.) } \pm 9 \text { (lumi.) pb. }
$$

This result is in good agreement with the theoretical prediction $\sigma_{t \bar{t}}^{t h e o r y}=238_{-24}^{+22} \mathrm{pb}$ for a top quark mass of $172.5 \mathrm{GeV}$ as obtained from approximate NNLO QCD calculations [7].

\section{Top pair relative differential cross-section measurement at $\sqrt{\mathrm{s}}=7 \mathrm{TeV}$ in the lepton+jets channel}

Since the predictions for the $t \bar{t}$ production cross-section are in very good agreement with the measurements, and considering the rapid increase of the integrated luminosity, the focus is starting

\footnotetext{
${ }^{1}$ The aplanarity $A$ is defined as $A=3 / 2 \lambda_{3}$, where $\lambda_{3}$ is the smallest eigenvalue of the normalized momentum tensor calculated using the momenta of all jets and the lepton.
} 
to switch to the "differential" measurements of the top properties, such as the cross-section as a function of the $t \bar{t}$ system kinematic variables. Such experimental measurements, performed in different channels, allow a precision test of the predictions of perturbative QCD. New physics may also give rise to additional $t \bar{t}$ production mechanisms or modifications of the top quark decay channels that can be discovered looking at the differential spectra, since the integrated cross-section is in good agreement with the SM prediction.

The ATLAS experiment has measured the relative $t \bar{t}$ production cross-section $\frac{1}{\sigma} \frac{d \sigma}{d X}$ as a function of the mass, transverse momentum and rapidity of the system at $\sqrt{s}=7 \mathrm{TeV}$ with $2.05 \mathrm{fb}^{-1}$ of data [9]. The topology under study is the single-lepton channel. The analysis has been performed using a cut-based approach: events have been selected in order to reduce the background contribution and enhance the signal-over-background ratio.

The $t \bar{t}$ system reconstruction is performed through a kinematic fit using a maximum likelihood approach. The likelihood assesses the compatibility of the event with a typical $t \bar{t}$ pair. The algorithm takes as input up to 5 reconstructed highest- $p_{T}$ jets and their and the information on the probability that the jet originates from a $b$ quark ( $b$-taggging), the lepton and the $E_{T}^{\text {miss }}$, using as fixed parameters the mass and the widths of the top and the $W$ boson. The $t \bar{t}$ system is then defined as the permutation of the four jets, lepton and $E_{T}^{\text {miss }}$ that maximizes the likelihood.

Once the reconstructed kinematic distributions are extracted, the cross-section is calculated in the full phase space at the parton level via unfolding methods. The measurements have been compared to NLO predictions from MCFM [8] for all variables and to NLO+NNLL [10] predictions for $m_{t \bar{t}}$. All measurements show good agreement with the theory, as shown in Fig. 2.

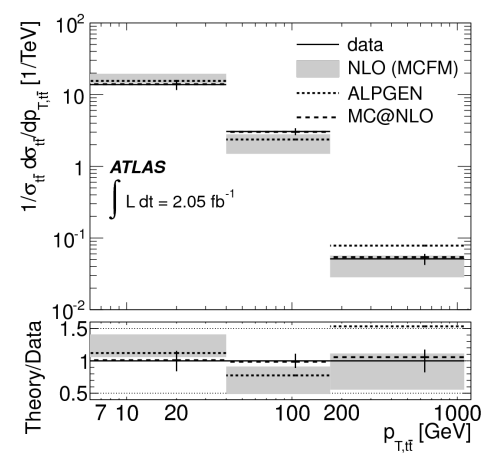

(a)

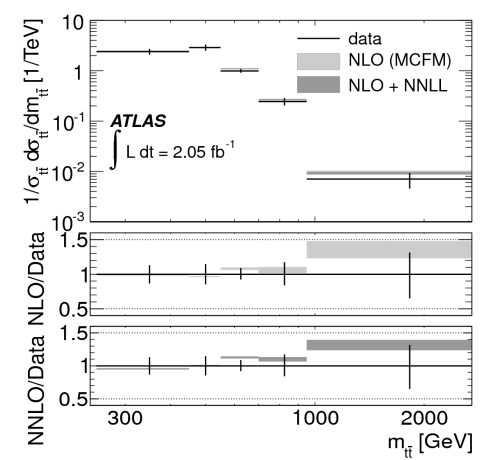

(b)

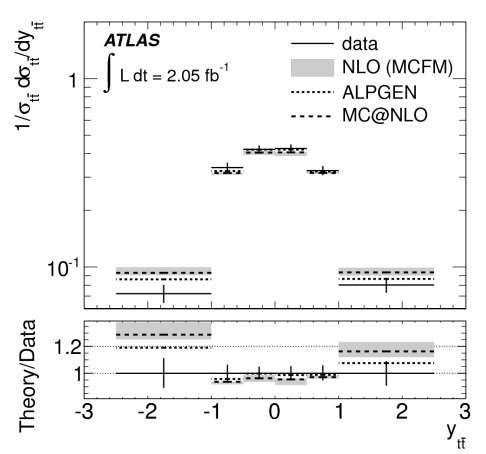

(c)

Figure 2: Relative differential $t \bar{t}$ cross-section at $\sqrt{s}=7 \mathrm{TeV}$ as a function of the transverse momentum (a), mass (b) and rapidity (c) of the $t \bar{t}$ system.

\section{Single top cross-section measurements at $\sqrt{\mathrm{s}}=\mathbf{7 \mathrm { TeV }}$}

Top quarks can be produced individually in electroweak interactions in $p p$ and $p \bar{p}$ collisions. Three different mechanisms contribute to single top-quark production: through the exchange of a time-like ( $s$-channel) and space-like ( $t$-channel) $W$ boson, and the production of a single topquark in association with an on-shell $W$ boson (Wt-channel). At the LHC, the $t$-channel production mechanism dominates, followed by the $W t$-channel, while the $s$-channel contribution is the lowest one.

At $\sqrt{s}=7 \mathrm{TeV}$ ATLAS has performed single top-quark cross-section measurements in the $t$ $[11,12], W t$ - and $s$-channel $[13,14]$. In particular, evidence for single top-quark production in the 
$W t$-channel has been observed for the first time at LHC (at a level of 3.3 $\sigma$ ), while for the $s$-channel production, only an upper limit has been measured.

A summary of the single top-quark cross-section at $\sqrt{s}=7 \mathrm{TeV}$ is shown in Fig. 3a. All measurements are in good agreement with the NLO+NNLL predictions [15-17].

\section{Single top cross-section measurement at $\sqrt{\mathbf{s}}=8 \mathrm{TeV}$ in the spacelike production channel}

At $\sqrt{s}=8 \mathrm{TeV}$ the single-top quark production cross-section has been measured in the $t$-channel production mechanism using the lepton+jets decay topology [18].

To separate $t$-channel single top-quark signal events from the expected background, several kinematic variables have been combined into one discriminant by employing a neural network (NN). For the training of the NN only the MC simulated events are used. Background from multijet QCD events are not used in the network training. The training of the NNs is done independently in the two-jet and three-jet samples.

A binned maximum-likelihood fit to the NN output distribution is performed simultaneously for the two-jet and three-jet samples to extract the $t$-channel single top-quark cross secion. The rates of the background processes have also been fitted within their uncertainties.

The cross-section of $t$-channel single top-quark production is measured to be

$$
\sigma_{t}(t)=95.1 \pm 2.4(\text { stat. }) \pm 18.0 \text { (syst.) } \mathrm{pb}=95.1 \pm 18.1 \mathrm{pb}
$$

which is in very good agreement with the SM prediction, as shown in Fig. 3 b.

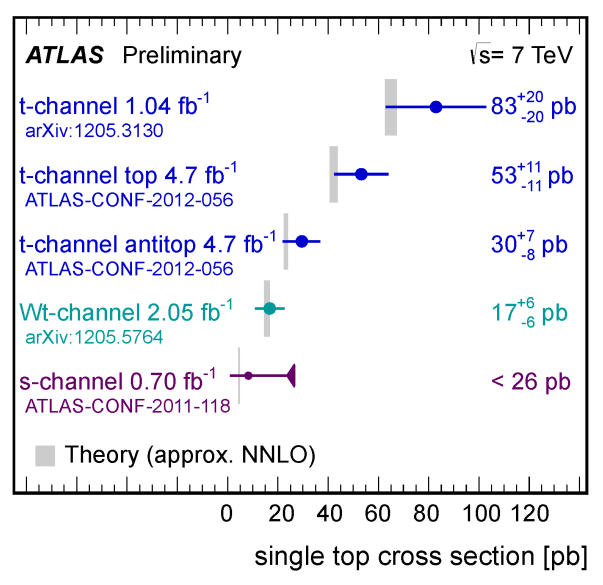

(a)

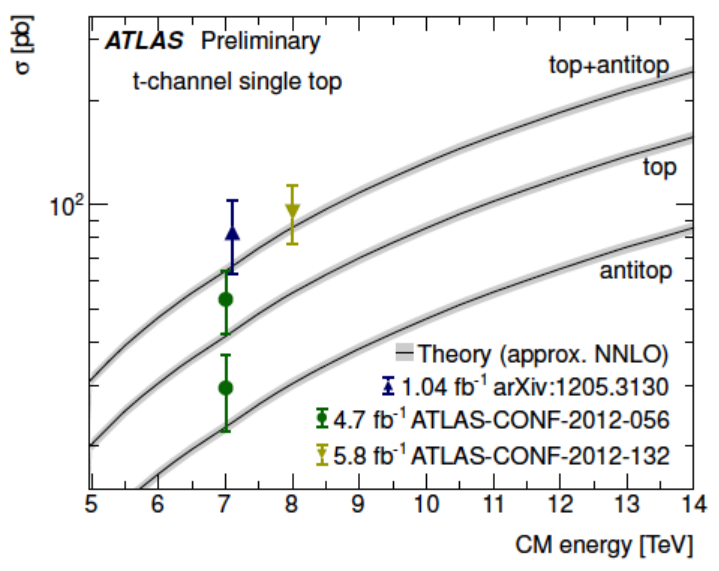

(b)

Figure 3: (a): summary of single top cross-section measurements at $\sqrt{s}=7 \mathrm{TeV}$. (b): compilation of ATLAS single top $t$-channel cross-section measurements at $\sqrt{s}=7 \mathrm{TeV}$ and $8 \mathrm{TeV}$.

\section{Top mass measurements}

The top quark mass, $m_{t}$, is a free parameter of the SM and must be determined experimentally. A precise determination of $m_{t}$ is crucial since quantum loops including top quarks induce large 


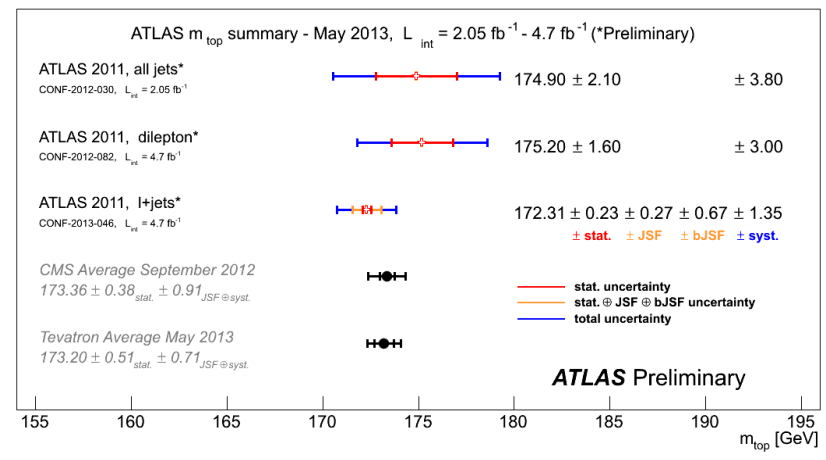

Figure 4: Summary of top mass measurements.

corrections to theory predictions for many precision electroweak observables, including the mass of the Higgs boson.

The most precise measurement at the moment has been performed in the single lepton channel with $4.7 \mathrm{fb}^{-1}$ of data at $\sqrt{s}=7 \mathrm{TeV}$ [19]. This measurement has been performed using, for the first time in ATLAS, a 3-dimensional template technique which determines the top quark mass together with a global jet energy scale factor (JSF), and a relative b-jet to light-jet energy scale factor (bJSF), where light jets refers to u-, d-, c-, s-quark jets. Templates are built for three observables:

- the reconstructed top-quark mass $m_{t}^{r e c o}$, obtained with the kinematic likelihood fit described in Sect. 3 in which $m_{t}$ and $m_{W}$ are considered fit parameters;

- the invariant mass of the hadronically decaying $\mathrm{W}$ boson, $m_{W}^{r e c o}$, calculated from the selected jet permutation;

- an observable called $R_{l b}^{r e c o}$ computed as the ratio between the transverse momentum of the $\mathrm{b}$-tagged jet and the average transverse momentum of the two light jets from the hadronic $\mathrm{W}$ boson decay for events with one b-jet or, for events with two or more b-jets, as the ratio of the scalar sum of the transverse momenta of the b-tagged jets assigned to the decaying top quarks and the scalar sum of the transverse momenta of the two light jets of the hadronic $W$ boson decay.

To determine simultaneously a global jet energy scale factor (JSF), a relative b-jet to light-jet energy scale factor (bJSF) and a top quark mass, templates of $m_{t}^{\text {reco }}$ are constructed as a function of the top quark mass used in the Monte Carlo generation in the range $167.5-177.5 \mathrm{GeV}$, for an input value of JSF in the range $0.95-1.05$ and for an input value of bJSF in the same range. Separate Monte Carlo samples are used for the different mass points, and templates with different values of bJSF and JSF are extracted from each by scaling the energies of the jets in each sample appropriately. Similarly, templates of $m_{W}^{r e c o}$ are constructed as functions of an input JSF, while templates of $R_{l b}^{\text {reco }}$ as functions of an input bJSF and $m_{t}$, using the same ranges as above. All templates are fitted with pre-determined probability density functions (PDFs): fits are done separately for events with one b-tagged jet, and for events with at least two b-tagged jets, to exploit the different sensitivities to the underlying $m_{t}$, JSF and bJSF. These PDFs are used in an unbinned likelihood fit to the data for all events, with a likelihood defined as

$$
L\left(m_{t}^{\text {reco }}, m_{W}^{\text {reco }}, L_{l b}^{\text {reco }} \mid m_{t}, J S F, b J S F, n_{b k g}\right) .
$$


The result for the top mass extracted from the fit is then:

$$
m_{t}=172.31 \pm 0.75(\mathrm{stat}+\mathrm{JSF}+\mathrm{bJSF}) \pm 1.35(\mathrm{syst}) \mathrm{GeV} .
$$

The simultaneous fit of the JSF and bJSF, that are the most important sources of uncertainty in these measurements, results in a significant reduction of the systematic uncertainty, at the cost of a small increase of the statistical uncertainty, due to the high dimensionality of the fit.

\section{Conclusions}

The study of top quarks can answer fundamental questions on the SM. This quark is undoubtedly a peculiar particle, with a Yukawa coupling of the order of one and it is a powerful probe of physics beyond the SM. Its production mechanism in $p p$ collisions is described very well by the SM, as well as its decay.

All the measurements shown in this paper are limited by systematic uncertainties. As more data is being analyzed our understanding of the detector is improving and calibrations are being refined. ATLAS is looking forward to refine the current measurements, as well as to produce new results using the full statistics recorded in 2012.

\section{References}

[1] F. Abe et alii, Phys. Rev. Lett 8 (74) 2626 [hep-ex/9503002].

[2] S. Abachi et alii, Phys. Rev. Lett. 8 (74) 2632 [hep-ex/9503003].

[3] https://twiki.cern.ch/twiki/bin/view/AtlasPublic/TopPublicResults.

[4] ATLAS Collaboration, JINST 3 S08003.

[5] ATLAS Collaboration, ATLAS-CONF-2012-024, http://cds.cern.ch/record/1430733.

[6] ATLAS Collaboration, ATLAS-CONF-2012-149, http://cds.cern.ch/record/1493488.

[7] M. Aliev et alii, Comput. Phys. Commun. 18210341046 [arXiv: 1007.1327 ].

[8] MCFM - Monte Carlo for FeMtobarn processes. http://mcfm.fnal.gov/.

[9] ATLAS Collaboration, Eur. Phys. J. C 732261.

[10] V. Ahrens et alii, J. High Energy Phys. 1009097 [arXiv:1003.5827 [hep-ph] ].

[11] ATLAS Collaboration, Phys. Lett. B 71733 [arXiv:1205.3130 [hep-ex]].

[12] ATLAS Collaboration, ATLAS-CONF-2012-056, http://cds.cern.ch/record/1453783.

[13] ATLAS Collaboration, Phys. Lett. B 716142 [arXiv:1205.5764 [hep-ex]].

[14] ATLAS Collaboration, ATLAS-CONF-2011-118, http://cds.cern.ch/record/1376410.

[15] N. Kidonakis, Phys. Rev. D 83091503 [arXiv:1103.2792 [hep-ph] ].

[16] N. Kidonakis, Phys. Rev. D 81054028 [arXiv: 1001.5034 [hep-ph]].

[17] N. Kidonakis, Phys. Rev. D 82054018 [arXiv: 1005.4451 [hep-ph] ].

[18] ATLAS Collaboration, ATLAS-CONF-2012-132, http://cds.cern.ch/record/1478371.

[19] ATLAS Collaboration, ATLAS-CONF-2013-046, http://cds.cern.ch/record/1547327. 